\title{
A sensitivity analysis on the springback behavior of the Unconstrained Bending Problem
}

\author{
T. Meinders ${ }^{1,2}$, A.W.A. Konter ${ }^{1}$, S.E. Meijers ${ }^{1}$, E.H. Atzema ${ }^{3}$, H. \\ Kappert $^{4}$
}

1 Netherlands Institute for Metals Research, Rotterdamseweg137, 2628 AL Delft, The Netherlands

2 University of Twente, Faculty of Engineering Technology, P.O. Box 217, 7500 AE Enschede, The Netherlands

3 Corus Research Development \& Technology, IJTC, Automotive Applications, P.O. Box 10000, 1970 CA IJmuiden, The Netherlands

4 Polynorm, Amersfoortseweg 9, 3751LJ, Bunschoten, The Netherlandsetherlands Institute for Metals Research - P.O. Box 5008, 2600 GA DELFT, Netherlands

e-mail:V.T.Meinders@ctw.utwente.nl

\begin{abstract}
Sheet metal forming software is commonly used in the automotive and sheet metal sectors to support the design stage. However, the ability of the currently available software to accurately predict springback is limited. A sensitivity analysis of the springback behavior of a simple product is performed to gain more knowledge into the various factors contributing to the predictability of springback. The sensitivity analysis comprises both numerical and physical aspects and results are reported in this article.
\end{abstract}

KEY WORDS: springback, sensitivity analysis, sheet metal forming 


\section{Introduction}

In the automotive industry, guidelines and finite element software are used in the design process of new sheet metal parts. Currently, the accuracy and reliability of these guidelines and numerical simulations do not satisfy the industrial requirements. The main limitation is the lack of an accurate springback prediction. To gain more knowledge and a better insight into the various factors contributing to the predictability of springback, a sensitivity analysis will be performed on a simple product, i.e. the unconstrained bending problem. The unconstrained bending problem served as a benchmark problem at the $5^{\text {th }}$ International Conference and Workshop on Numerical Simulation of 3D Sheet forming Processes (Numisheet 2002). Although the authors are aware that this benchmark is not representative of sheet metal forming in general, this problem was chosen since it shows severe springback after forming, which enables a clear sensitivity analysis (unfortunately, in general, the causes for differences in the benchmark results are not discussed within the benchmark study, as was the case for this benchmark). The performed sensitivity analysis comprises both numerical and physical factors and is carried out using four different software packages, i.e. Abaqus/Standard, MSC.MARC, Optris and Dieka. The article starts with a description of the benchmark specification and a short overview of the results of the Numisheet 2002 benchmark participants. Next, the results of the sensitivity analysis will be discussed. Finally, conclusions are drawn and recommendations are given.

\section{Numisheet 2002 Unconstrained bending problem}

\subsection{Benchmark specification}

The geometrical layout of the unconstrained cylindrical problem as proposed for the Numisheet 2002 conference (Numisheet 2002) is shown in Figure 1. The total punch stroke is $28.5 \mathrm{~mm}$, meaning that the punch stops moving when the punch and die have become concentric, see Figure 2. All tools are composed of hardened tool steel with a surface roughness of less than $\mathrm{Ra}=5 \mu \mathrm{m}$. A constant punch speed between $1 \mathrm{~mm} / \mathrm{s}$ and $50 \mathrm{~mm} / \mathrm{s}$ is prescribed. The geometrical parameters of the problem are summarized in Table 1. Both aluminum and high strength steel were used in this benchmark. For material specification and related lubrication specifications, the reader is referred to Numisheet 2002. Note that the experiments were to be conducted in as-received condition. 


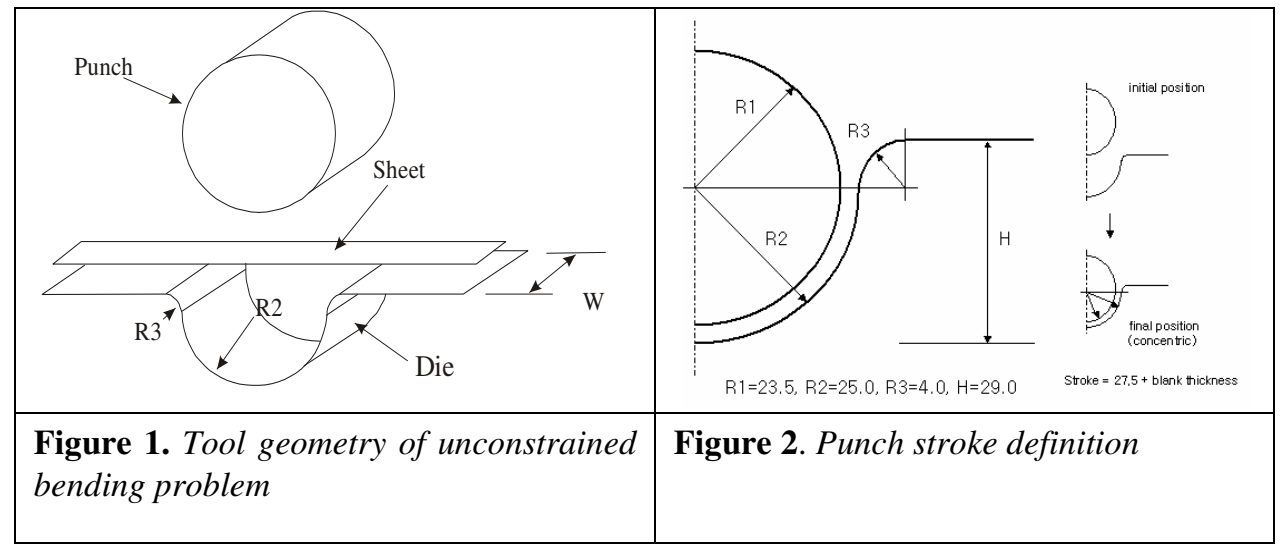

\begin{tabular}{|c|c|c|c|}
\hline Model parameters & $(\mathrm{mm})$ & Model parameters & $(\mathrm{mm})$ \\
\hline Punch radius & 23.5 & Length of sheet (initially) & 120.0 \\
\hline Die radius R2 & 25.0 & Thickness of sheet & 1.0 \\
\hline Die shoulder R3 & 4.0 & Width of sheet & 30.0 \\
\hline Width of tools & 50.0 & Punch stroke & 28.5 \\
\hline
\end{tabular}

Table 1. Geometrical parameters

To measure springback, angle "ABDC" as defined in Figure 3, is used. This angle is measured after forming at the maximum punch displacement and after springback. The difference between these angles quantifies the actual springback.

\subsection{Benchmark results}

This section gives a short overview of the results presented at the Numisheet 2002 conference Numisheet 2002. The unconstrained bending test is performed on test specimens cut at $0^{\circ}, 45^{\circ}$ and $90^{\circ}$ from the rolling direction, for both aluminum and high strength steel. Experiments were performed by 4 participants (BE-01 till BE-04), simulations were performed by 18 participants (BS-01 till BS-18). The participants used several FE codes, while some FE codes were used by several participants. Concerning the simulation results, the focus will be on participant BS-18 ('University of Twente, the Netherlands', using the finite element code Dieka) since this participant contributed also to the sensitivity analysis as described in this article. Here, only the results for the rolling direction will be discussed. The discussion will however be brief, since the Numisheet 2002 benchmark results will only serve as an illustration in the paper at hand. 


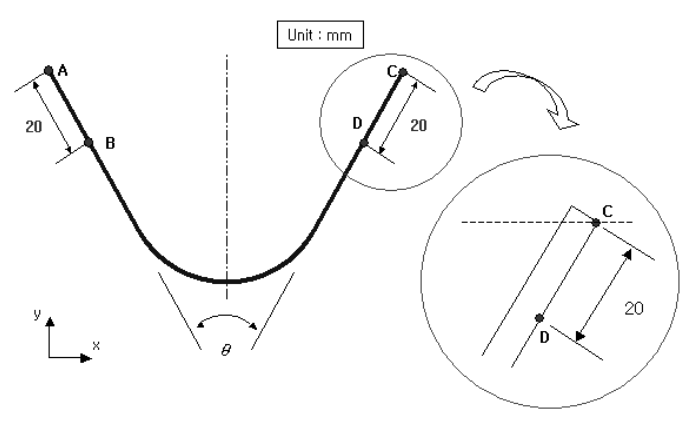

Figure 3. Definition of angle to measure springback

Figure 4 and Figure 5 give the angles after forming and after springback for aluminum sheets. The horizontal axis represents the participants, the vertical axis the corresponding angle. The grey bar in the graphs depicts the bandwidth of the experimental results (first 4 dots in the graphs are experiments). For both the experiments and simulations, the arithmetic mean $(\mu)$ and standard deviation $(\sigma)$, based on a normal distribution, are given in the subscript of the figures. Figure 6 and Figure 7 give the angle after forming and after springback for high strength steel. Figure 8 and Figure 10 give the experimental force- displacement curves for both the aluminum and high strength steel. Figure 9 and Figure 11 give the numerical results of the forcedisplacement curves for both the aluminum and high strength steel of all participants. The contribution of participant BS-18 is marked with solid squares and the experimental results of participant BE-02 ('laboratoire Génie Mécanique et Matérieaux, France'), marked with solid circles, are added for comparison.

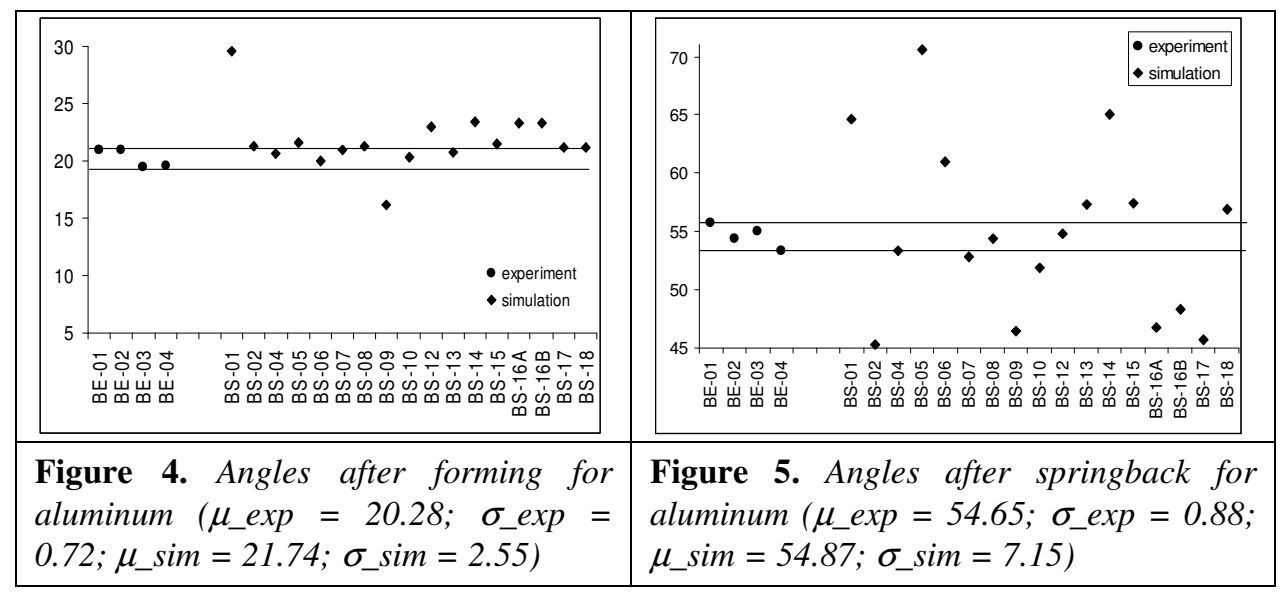




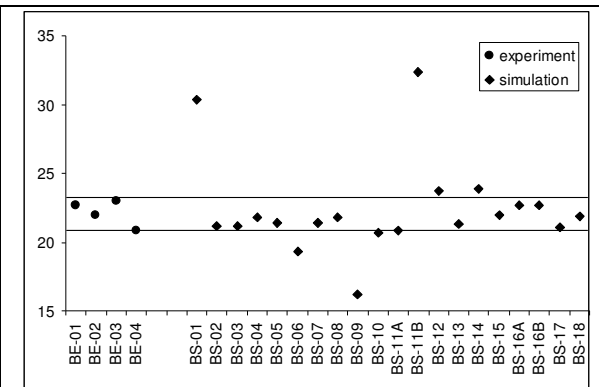

Figure 6. Angles after forming for high strength steel $\left(\mu \_\exp =22.13 ; \sigma \_\exp =\right.$ $0.85 ; \mu \_s i m=22.40 ; \sigma \_s i m=3.40$ )

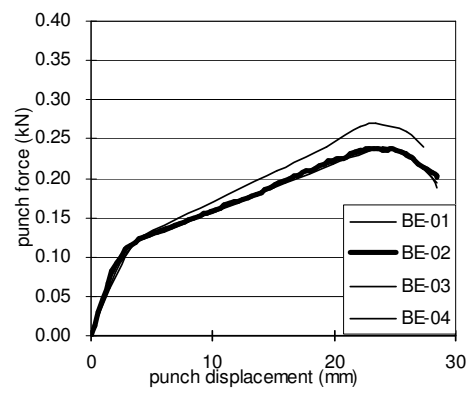

Figure 8. Exp. results: Punch forcedisplacement curve for aluminum

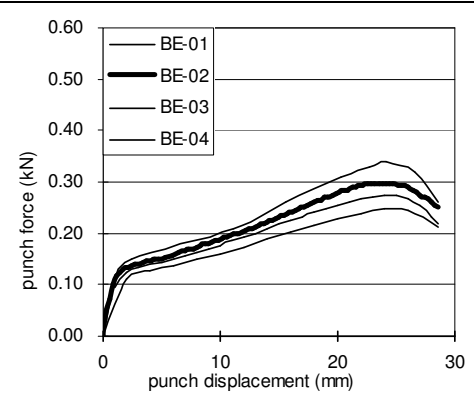

Figure 10. Exp.results: Punch force displacement curve for HSS

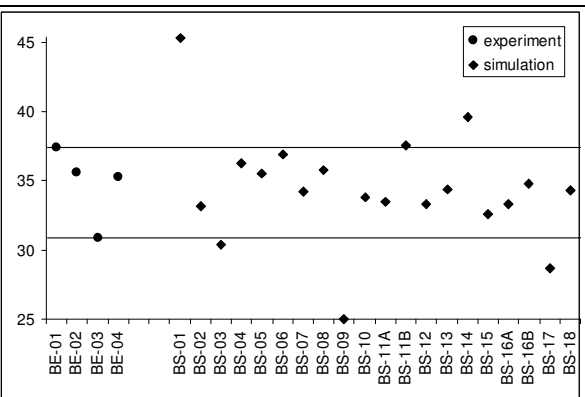

Figure 7. Angles after springback for high strength steel $\left(\mu \_\right.$exp $=34.80 ; \sigma \_$exp $=$ $\left.2.39 ; \mu \_\operatorname{sim}=34.43 ; \sigma \_\operatorname{sim}=3.97\right)$

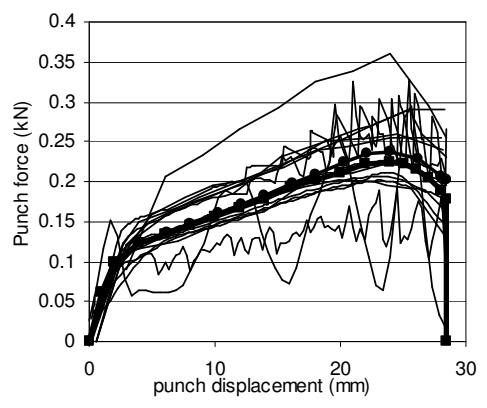

Figure 9. Numerical results: Punch forcedisplacement curve for aluminum

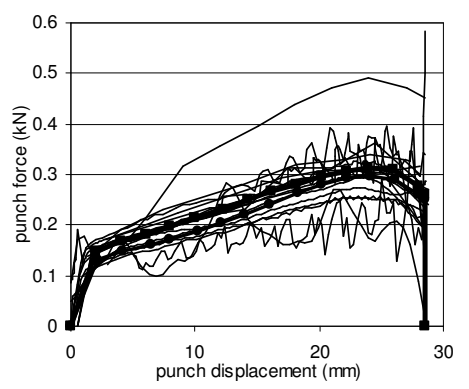

Figure 11. Num.results: Punch force displacement curve for HSS 


\subsection{Discussion on benchmark results}

\subsubsection{Load displacement curve}

Experimental results for aluminum:

The scatter in the results, obtained by three of the participants is negligible. The forces indicated with BE-03 are consistently about $15 \%$ higher than those presented in the curves BE-01, BE-02 and BE-04. This is likely to be a systematic error, rather than noise.

\section{Experimental results for high strength steel:}

The scatter in the results ranges from $0.25 \mathrm{kN}$ (BE-01) to $0.34 \mathrm{kN}$ (BE-03). The results of participants BE-02 and BE-04 are more or less in the middle.

Numerical results for aluminum and high strength steel:

A remarkably large scatter occurs in the results of all participants for which the reason can be twofold. It is very likely that large oscillations in some of the results are caused by the tool speed settings in combination with using explicit time integration. Another reason for some of the oscillations is likely caused by tight contact settings in combination with localized contact. One of the results shows a maximum force, which is almost 2 times as high as the other results. This is either caused by tool speed or by too a strong damping technique. Some results show an initially smooth behavior and an instable behavior at the end of the load displacement diagram. This is caused by the fact that in the simulation only a few nodes are in contact. It is also noticed that the results of certain participants, which show a large deviation in case of aluminum reproduce this deviation in case of high strength steel.

\subsubsection{Angle after forming and after springback}

Experimental results for aluminum:

The angles after forming differ about $1.5^{\circ}$ in the reported results. A maximum difference of $2.5^{\circ}$ is present in the angle after springback.

Experimental results for high strength steel:

The angles after forming differ about $2^{\circ}$ in the reported results. A maximum difference of $6.5^{\circ}$ is present in the angle after springback.

Numerical results for aluminum and high strength steel:

A large scatter appears in the predicted angles after forming. However, since the displacements of the tools are prescribed, one would expect that the predicted angles at the end of the forming stage have to be almost identical. Most likely, a wrong choice of contact stiffness and/or damping technique causes this large scatter, as will be illustrated in sections 4.1, 4.2 and 4.3. Incorrect contact checking probably causes the predicted 
angle deviation of approximately $16^{\circ}$ between highest and lowest simulation result. Even more scatter appears in the angle after springback, due to the above-mentioned reasons. Nevertheless, five results of implicit codes, two results of explicit codes and one result of an implicit/explicit code are within (or close to) the range of the experimental results. Hence, from the presented results, it cannot be concluded that either explicit, explicit/implicit or implicit techniques provide more accurate answers for the springback problem.

\subsubsection{Conclusion}

The scatter in the experimental results in case of aluminum is low. The scatter in the experimental results in case of high strength steel is somewhat higher but still acceptable. From an experimental point of view, this unconstrained bending problem is well defined, so probably measuring errors and fluctuation of the material properties of the test specimens cause the scatter in the data. The scatter in the numerical results of the Numisheet 2002 benchmark is unrealistically large. The reason for this can be numerous, like:

- Difficulties caused by local sliding contact, which is difficult to treat in a discrete manner

- Applied punch speed in case of using explicit codes

- Applied stabilization techniques in contact treatment, like contact stiffness, hard or soft contact, damping techniques etc.

- Applied tool discretization versus an analytical description of the tool geometry.

- Applied material models, comprising yield functions (Von Mises, Hill, Barlat, Vegter) and hardening models (isotropic / kinematic hardening)

- The influence of inexperienced users.

The results of participant BS-18 are in good agreement with the experimental results for both aluminum and high strength steel. This can be observed for both the load displacement diagrams and the predicted angles after forming and after springback. Note that although participants BE-03 ('Corus, the Netherlands') and BS-18 do have cooperation, the sended benchmark results were achieved seperately so as not to undermine the benchmark idea. The results of BS-18, as reported by the benchmark committee, would then obviously have been closer to BE-03 than to the average, as it is now. 


\section{Set up of the sensitivity analysis}

The current sensitivity analysis will be performed with four different finite element codes (Abaqus/Standard, MSC.MARC, Optris and Dieka) to exclude the possibility that specific code artifacts will turbid the analysis and to explore the possible consequence of present artefacts. This means that one has to be critical to what is to be compared. Therefore, it is chosen to define the default settings of the sensitivity analysis as general as possible. A mesh density with a typical element length of $2 \mathrm{~mm}$ is used. The material model is assumed isotropic with Nadai hardening. The material properties used are listed in Table 2.

\begin{tabular}{|l|l|l|l|}
\hline Elasticity modulus E & $70.5 \mathrm{Gpa}$ & Nadai, $C$ & $550.4 \mathrm{Mpa}$ \\
\hline Poisson ratio $v$ & 0.346 & Nadai, $n$ & 0.223 \\
\hline R0, R45, R90 & $1,1,1$ & Initial yield stress $\sigma_{\mathrm{y} 0}$ & $194.1 \mathrm{Mpa}$ \\
\hline
\end{tabular}

Table 2. Default material properties

However, several differences between de finite element codes remain present, which can cause differences in the simulation results:

- For Abaqus/Standard and Dieka a parameter combination with penalty stiffness of $200 \mathrm{MPa} / \mathrm{mm}$ is specified, using rigid tools. In MSC.MARC a deformable tool, combined with constraint equations is used. In Optris a rigid tool is specified and exact contact is achieved using a Lagrange multiplier technique.

- In Abaqus/Standard, MSC.MARC and Dieka an analytical tool description is used, while Optris uses a discretized tool with a typical element length of $0.22 \mathrm{~mm}$.

- In Abaqus/Standard a 4-noded shell with reduced integration (type S4R) is applied, In Dieka, the discrete shear triangle is used (Batoz J.L et al.,1989). Optris makes use of a 4-noded Belytschko element, comprising bilinear interpolation and satisfying the Mindlin Reissner assumptions (Optris,2002). In MSC.MARC a 4noded thick shell element is used (MSC.MARC, 2002).

- With Abaqus/Standard, Dieka and MSC.MARC an implicit solution technology is applied, while Optris uses an explicit solution technology.

- Optris and Dieka make use of an instantaneous release of the tools, whereas a virtual retraction of the tools is applied in Abaqus/Standard and MSC.MARC.

With these default settings, the computation time on a HP8000 workstation for a simulation with default settings was around 2.5 hours for a shell analysis and around 1 hour for a plane strain analysis.

To investigate how the particular finite element solutions compare to each other for the default setting, two sets of simulations are performed, i.e. one set with a friction 
coefficient $(\mu)$ of 0.1348 (according specifications of (Numisheet 2002)) and one set without friction, see Figure 12 and Figure 13.

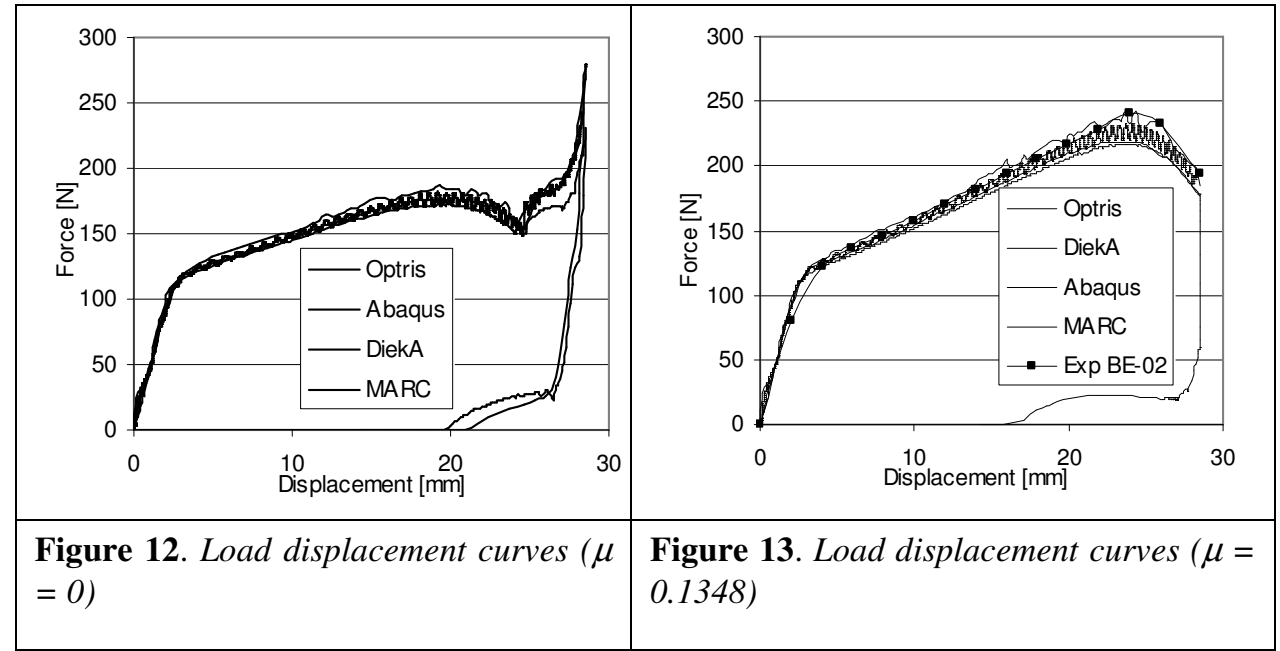

The results of the simulations with friction are qualitatively compared to the experimental results. Note that the results of the Numisheet 2002 benchmark and the results of the sensitivity analysis, to be discussed in this article, cannot be quantitatively related, since in most analyses in the sensitivity study isotropic material behavior is assumed. From Figure 12 and Figure 13 it is concluded that the results obtained using different programs and technologies are in good agreement with each other for the chosen default settings. Since the results of the simulations performed in the sensitivity analyses, to be described in the next sections, using Abaqus/Standard, MSC.MARC, Optris and Dieka, are quite close and since specific phenomena can be reproduced with more than one of these codes, it can be concluded that all simulations within the sensitivity analyses are performed sufficiently accurate to have a valuable and valid sensitivity analysis. Note that the scatter in the load-displacement curve for the results presented here is considerably lower than the results presented at Numisheet 2002.

\section{Sensitivity analysis: Numerical factors}

The driving factor in most metal forming analyses is the contact between tool and sheet. In a numerical simulation the tool is often considered as rigid and is either represented by an analytical surface or a discretized surface.

The contact behavior (in particular between the sheet and the punch) is very local in the current benchmark problem. The sheet is modeled with a number of elements. For 
these elements a boundary contour can be defined consisting of boundary nodes and boundary segments (2D) or boundary patches (3D). In a deformable-rigid contact description contact checking is performed only for the nodes of this boundary contour. The tool surface is modeled with a number of rigid segments. These surface segments can be either curved (analytical description) or straight (discrete description). Physically it can occur that contact between the sheet and the tool is only present at one segment of the sheet and one segment of the tool. Since numerically contact checking is only performed between the boundary nodes of the sheet and the surface of the tools it can occur that no contact is detected during a specific increment and hence a rigid body mode for the deforming sheet is present at some stage during the deformation history. If a penalty based method of contact is applied the contact between the sheet and the tools is modeled with springs and depending upon the applied penalty function value (i.e. value of the spring stiffness) the contact will be less local at the cost of some penetration of the sheet between the tool.

\subsection{Contact stiffness}

In order to determine contact, the current position of a node of the sheet relative to the position of the (discretized) tool surface is checked. The contact conditions in normal direction can be enforced in two ways: a) specification of a constraint on the displacement increments and b) by application of penalty stiffness between the node and surface. The accuracy of the latter method is determined by the numerical value of the penalty stiffness. Too small a value of the stiffness causes penetration of the surface, but has the benefit of a more smooth contact.

A parameter study on the effect of the penalty stiffness on the springback angle has been performed with Dieka and Abaqus/Standard, see Table 3 till Table 5 (3D-shell and 2D-plane strain formulation). The parameter study has been repeated for the simulation without friction with Abaqus/Standard, Table 6 and Table 7.

\begin{tabular}{|l|l|l|}
\hline Penalty stiffness $(\mathrm{MPa} / \mathrm{mm})$ & Forming angle & springback angle \\
\hline 100 & 22.68 & 56.91 \\
200 & 21.95 & 56.42 \\
300 & 21.63 & 56.13 \\
400 & 21.45 & 55.98 \\
500 & 21.33 & 55.88 \\
2500 & 20.73 & 55.45 \\
5000 & 20.60 & 55.33 \\
\hline
\end{tabular}

Table 3. Variation of penalty stiffness (Dieka; $\mu=0.1348 ;$ shell)

\begin{tabular}{|l|l|l|} 
Penalty & Forming angle & springback angle \\
\hline
\end{tabular}




\begin{tabular}{|l|l|l|}
\hline stiffness $(\mathrm{MPa} / \mathrm{mm})$ & & \\
\hline 200 & 21.83 & 53.89 \\
1000 & 20.92 & 53.27 \\
\hline
\end{tabular}

Table 4. Variation of penalty stiffness (Abaqus/Standard; $\mu=0.1348 ;$ shell)

\begin{tabular}{|l|c|c|}
\hline $\begin{array}{l}\text { Penalty } \\
\text { stiffness }(\mathrm{MPa} / \mathrm{mm})\end{array}$ & Forming angle & springback angle \\
\hline 200 & 21.84 & 54.45 \\
500 & 21.19 & 53.88 \\
1000 & 20.90 & 53.55 \\
5000 & 20.56 & 53.30 \\
\hline
\end{tabular}

Table 5. Variation of penalty stiffness (Abaqus/Standard; $\mu=0.1348$; plane strain)

\begin{tabular}{|c|l|l|}
\hline Penalty stiffness $(\mathrm{MPa} / \mathrm{mm})$ & Forming angle & springback angle \\
\hline 200 & 21.81 & 44.22 \\
1000 & 20.89 & 43.30 \\
\hline
\end{tabular}

Table 6. Variation of penalty stiffness (Abaqus/Standard; $\mu=0.0 ;$ shell)

\begin{tabular}{|l|l|l|}
\hline Penalty stiffness $(\mathrm{MPa} / \mathrm{mm})$ & Forming angle & springback angle \\
\hline 200 & 21.88 & 48.38 \\
500 & 21.24 & 47.89 \\
1000 & 20.94 & 47.69 \\
5000 & 20.61 & 47.57 \\
Hard & 20.45 & 49.02 \\
\hline
\end{tabular}

Table 7. Variation of penalty stiffness (Abaqus/Standard; $\mu=0.0$; plane strain)

The forming angles, predicted with both programs and the shell/plane strain formulation, are in good agreement for equivalent values of the penalty stiffness. Even for the high values of the penalty stiffness a converged value is not obtained, when compared to the so-called hard condition, see Table 7. Hard condition means applying constraints on the displacements.

\section{Analyses with friction}

A difference of maximum $2.0^{\circ}$ in forming angle is obtained depending upon the penalty stiffness; the maximum difference reads $1.6^{\circ}$ for the angle after springback. Note that angles after springback obtained with Dieka are typically $1.5^{\circ}$ larger than those obtained with Abaqus/Standard. This can easily be explained by the difference in tool release method, see section 4.7 .

\section{Analyses without friction}

The predicted angles after forming hardly differ from those with friction, the maximum difference in angle after springback is $0.8^{\circ}$. Note, that the angles after springback are considerably less compared to the analyses with friction, while a large 
difference is observed between the results obtained with the plane strain formulation and the shell formulation. This difference is explained in section 5.1.

\subsection{Rigid vs. deformable die}

In several finite element codes, like MSC.MARC, the non-penetration condition with respect to contact is strict, meaning that no overlap of contacting bodies is allowed. This type of contact representation is called 'hard contact'. In contrast to the penalty-based methods, no finite stiffness between contacting surfaces has to be provided. A consequence of this approach is that in metal forming applications like bending, the contact spots tend to be very small, i.e. a few nodes are actually touching, since the elastic properties of the tools and sheet are usually similar. Such localized contact may adversely affect the stability of a model, as any change in contact conditions can have a large effect. As a result the model may have difficulty to converge. In case of a sliding contact situation, the sensitivity to localized contact is significant. In the bending problem at hand, such a sliding contact situation exists near the die shoulder. The first attempts to simulate the bending, employing rigid bodies to represent the tools, therefore proved to be unsuccessful. Only after the number of increments had been increased to one thousand and the convergence controls had been drastically tightened, the first simulation was completed successfully. However the results appear questionable after inspection of the force-displacement curve, see Figure 14.

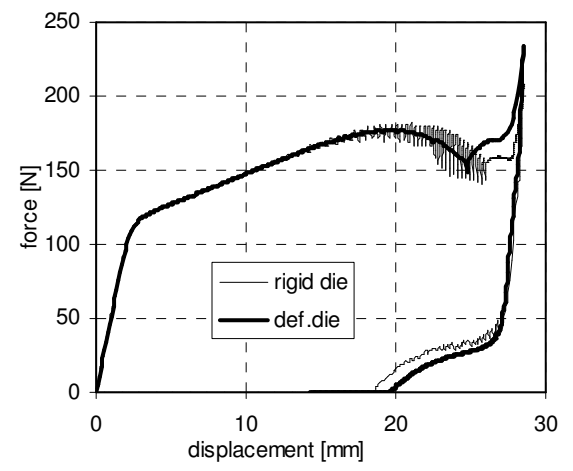

Figure 14. Punch force-displacement curve for rigid and deformable dies $(E=100$. GPa). (MSC.MARC; $\mu=0.0$; plane strain)

It is observed that the curve contains an almost periodic disturbance, whose amplitude gradually increases. Upon inspection, this ripple had to be attributed to the discrete representation of the sheet (as a regularly spaced set of nodes) sliding past the 
die radius. A local refinement of the mesh could help to reduce this ripple. In this way the discretization error can be reduced, but not removed. Even for a refined mesh the rapid oscillations of the force-displacement curve will remain, be it on a smaller scale. From a practical point of view, an extreme local mesh refinement could prove to be unattractive and/or computationally expensive. An alternative way to suppress the ripple is to model the die as a deformable contact body. By choosing a suitable contact body numbering (die first), it is thus possible to have nodes of the die touching the sheet instead of the other way around. The benefit of this approach is that a much more stable contact situation is obtained as the need for nodes to separate and the number of changing contact conditions in general, is much smaller. The force-displacement curve of this approach is also depicted in Figure 14. The simulation, using a deformable die, needs a total of 250 iterations, compared to at least a thousand for the rigid die. Note that the two curves in Figure 14 are somewhat different, especially near the maximum punch displacement. So, apart from the superimposed ripple, the discretization error in case of a rigid die also seems to affect the shape of the underlying force displacement curve. A possible explanation is that the sliding of the sheet is restrained, which leads to a different bending history of the sheet. To check if the results when using a deformable die are reasonable, the analysis of the rigid die case has been repeated with a locally refined mesh. A force-displacement curve is obtained which compares well to the case deformable die. The calculated forming and springback angles for both die types are listed in Table 8. Hence, it can be concluded that using a deformable tool gives reasonable results. In view of the extreme local mesh refinement necessary to limit the discretization errors in the rigid die approach, a deformable die representation is preferred.

\begin{tabular}{|l|l|l|}
\hline Die type & Forming angle & Springback angle \\
\hline rigid & 20.50 & 46.29 \\
Deformable & 20.48 & 45.89 \\
\hline
\end{tabular}

Table 8. Variation of die type (MSC.MARC; $\mu=0.0$; plane strain)

Next, the influence of the flexibility of the deformable die on the simulation results is investigated. The Young's modulus of the die is set to 1.0 GPa, 100.0 GPa and 10000.0 $\mathrm{GPa}$, respectively. The calculated forming and springback angles for the variation in Young's modulus are listed in Table 9.

\begin{tabular}{|l|c|c|}
\hline Young's modulus die $(\mathrm{GPa})$ & Forming angle & springback angle \\
\hline 1.0 & 22.16 & 48.00 \\
100.0 & 20.50 & 46.29 \\
10000.0 & 20.43 & 46.24 \\
\hline
\end{tabular}

Table 9. Variation of Young's modulus die (MSC.MARC; $\mu=0.0$; plane strain) 
Decreasing the Young's modulus from 100. GPa to 1.0 GPa largely affects the angle after forming (increase of $8 \%$ ). In case of using a modulus of $1.0 \mathrm{GPa}$, severe deformation of the die is observed, which influences the forming process significantly. When a modulus of $100.0 \mathrm{GPa}$ is used, hardly any deformation of the die is observed. Increasing the modulus from $100.0 \mathrm{GPa}$ to $10000.0 \mathrm{GPa}$ hardly affects the angles after forming and after springback (decrease of $0.3 \%$ ).

\subsection{Damping techniques}

In order to improve the convergence behavior of highly non-linear phenomena frequently numerical stabilization techniques are applied. The influence of stabilization techniques is investigated with both Abaqus/Standard and Dieka. First the results of the Abaqus/Standard simulations are discussed. This code provides an automatic mechanism for stabilizing unstable quasi-static problems through the addition of volumeproportional damping to the model. Viscous forces of the form $F_{v}=c M^{*} v$ are added to the global equilibrium equations, where $M^{*}$ is an artificial mass matrix calculated with unity density, $c$ is a damping factor, $v=\Delta u / \Delta t$ is the vector of nodal velocities and $\Delta t$ is the time increment. In the reference model a damping factor of $1.0 \mathrm{e}^{-7}$ has been applied, where after a set of simulations is performed in which the damping factor was decreased stepwise to $1.0 \mathrm{e}^{-3}$. The calculated forming and springback angles are listed in Table 10 . The characteristic load displacement curves are shown in Figure 15 for different values of the damping factor. Besides, Figure 16 gives an overview of the global energy quantities in case of a damping fact of $1.0 \mathrm{e}^{-3}$ : work performed by the external forces; work dissipated by friction; internal energy; plastic strain energy and stabilization energy.

\begin{tabular}{|l|l|l|}
\hline Damping factor & Forming angle & springback angle \\
\hline $1.00 \mathrm{E}-7$ & 21.84 & 54.45 \\
$1.00 \mathrm{E}-6$ & 21.84 & 54.44 \\
$1.00 \mathrm{E}-5$ & 21.85 & 54.36 \\
$1.00 \mathrm{E}-4$ & 21.90 & 53.75 \\
$1.00 \mathrm{E}-3$ & 22.46 & 31.07 \\
\hline
\end{tabular}

Table 10. Variation of damping factor (Abaqus/Standard; $\mu=0.1348$; plane strain)

From these results, it can be concluded that for damping factors smaller than $1.0 \mathrm{e}^{-5}$ the effect of the stabilization on the accuracy of the springback angle and load displacement curve can be neglected. For higher values of the damping factor incorrect results are obtained, since the work dissipated by numerical damping cannot be neglected. However, the numerical value of the factor cannot easily be applied directly to other sheet forming simulations. Unfortunately for each new problem this factor has 
to be determined as the damping factor is a function of the mesh density and the material properties.

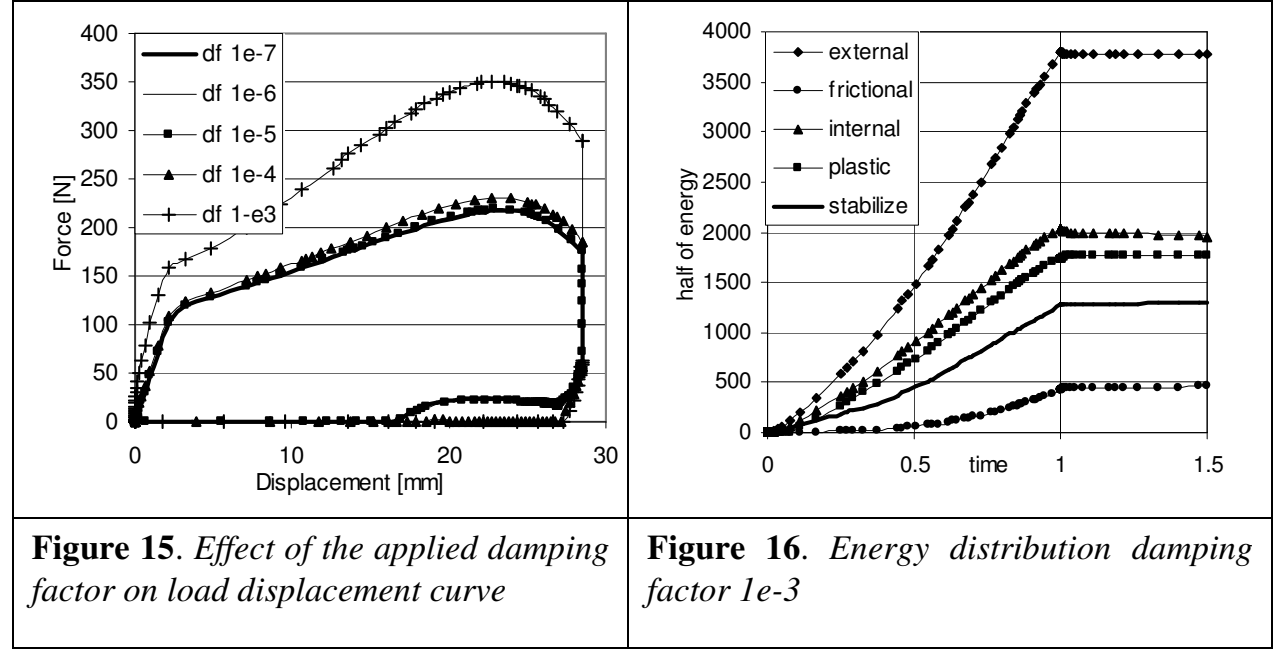

Next, the results of the Dieka simulations are discussed. In this code damping is applied by defining a damping length and a damping stiffness. The damping is a function of the change in gap function $g$. Damping starts to work at a value of gdamp. The damping stiffness is given by $C_{d}$. When $g>0$ (contact bodies are not in contact yet), the damping decreases with the reciprocal of its opening. Hence, the expression for the normal contact stress $\sigma_{n}$ looks like:

$$
\begin{aligned}
\sigma_{n} & =0 & \text { for } & g_{d}<g \\
& =\left(1-\frac{g}{g_{d}}\right) C_{d} \Delta g & \text { for } & 0<g \leq g_{d} \\
& =E_{c} g+C_{d} \Delta g & \text { for } & g \leq 0
\end{aligned}
$$

With, $\Delta g$ the change in gap function, $g_{d}$ the distance at which damping is activated and $E_{c}$ the normal contact stiffness. In the reference model, the damping distance was set to zero. A set of simulations is performed in which the damping distance is increased for a given damping stiffness (default is $0.3 *$ contact stiffness). The calculated forming and springback angles are listed in Table 11. 


\begin{tabular}{|l|c|c|}
\hline damping distance $(\mathrm{mm})$ & Forming angle & springback angle \\
\hline 0.0 & 22.06 & 56.42 \\
0.01 & 21.96 & 56.45 \\
0.1 & 22.02 & 56.67 \\
$>0.1$ & Not appl. & Not Appl. \\
\hline
\end{tabular}

Table 11. Variation of damping distance (Dieka; $\mu=0.1348 ;$ shell)

From these results it can be concluded that by introducing some amount of damping (damping distance $>0.1$ ) the simulation results already deteriorates the simulation, i.e. the sheet sticks to the die and as a result a complete different deformation pattern is achieved, see Figure 17.
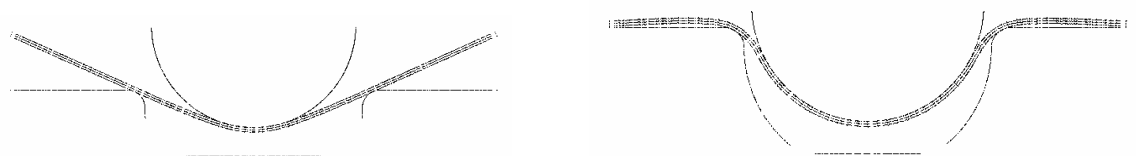

Figure 17. Deformed shapes without (upper) and with (lower) damping (damping distance $>0.1 \mathrm{~mm}$ )

\subsection{Tool discretization}

The tool geometry is simple and can easily be described with analytical surfaces. However, frequently tools are approximated by discretized surfaces. The number of elements used in this discretization affects the accuracy and the numerical solution process (contact distance and smoothness of the normal along the surface). In this section the analytical tool description will be compared to the discretized tool discretization. Three simulations are performed using a plane strain formulation without friction. First, the tool radius is described with a mesh using 20 elements along the tool radius, second a mesh using 150 elements along the tool radius is applied and finally an analytical description of the tool is used. Besides, a simulation is tried in which the tool radius is described with 10 elements. Unfortunately, this simulation did not converge during the springback stage. Therefore, the recorded angles of this simulation are not treated here. The simulation results are listed in Table 12 and the load displacement diagrams are shown in Figure 18.

\begin{tabular}{|l|l|l|}
\hline tool radius & Forming angle & springback angle \\
\hline Analytical & 21.88 & 48.51 \\
150 elms. & 21.88 & 48.38 \\
20 elms. & 22.19 & 48.80 \\
\hline
\end{tabular}

Table 12. Variation of tool discretization (Abaqus/Standard; $\mu=0.0 ;$ plane strain) 


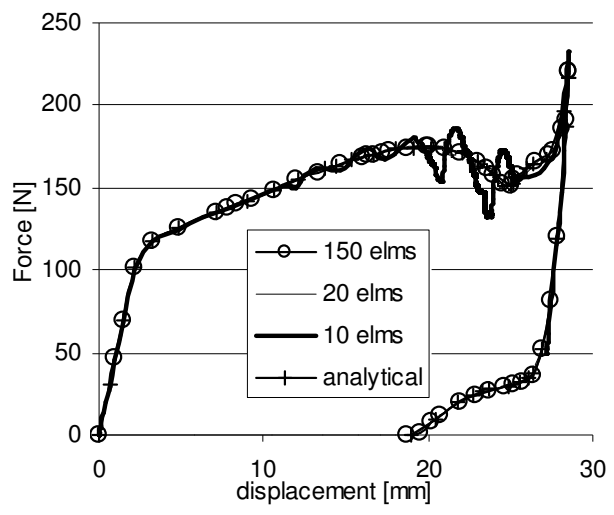

Figure 18. Load displacement curve for various tool discretization

It can be concluded that the applied mesh density of the tool radius does not influence the simulation results significantly. However, the convergence behavior is extremely sensitive to the local contact behavior. Contact checking is only performed for the nodes of the sheet with the tool. If a too coarse mesh is applied oscillations in the load displacement curve are present, see Figure 18 in case of using 10 elements over the tool radius. The availability of an analytical surface representation is clearly preferred. This conclusion is supported when looking at the required number of incremental steps during simulation, see Table 13.

\begin{tabular}{|l|l|l|}
\hline tool radius & Forming & Springback \\
\hline analytical & 133 & 25 \\
150 elms. & 377 & 26 \\
20 elms. & 734 & 37 \\
10 elms. & 3713 & not appl. \\
\hline
\end{tabular}

Table 13. Number of increments required for different tool radius descriptions

\subsection{Sheet discretization}

The sheet geometry can be meshed both in regular and irregular ways. A high mesh density is required to accurately describe the contact behavior between nodes and tools.

\subsubsection{Irregular meshes}

Two different mesh densities have been applied based on an irregular mesh. Resulting characteristic angles are shown in Table 9. 


\begin{tabular}{|c|c|c|}
\hline Element size $(\mathrm{mm})$ & Forming angle & springback angle \\
\hline 2.0 & 21.95 & 56.42 \\
1.2 & 21.74 & 56.68 \\
\hline
\end{tabular}

Table 14. Variation of element size (Dieka; $\mu=0.1348$; shell)
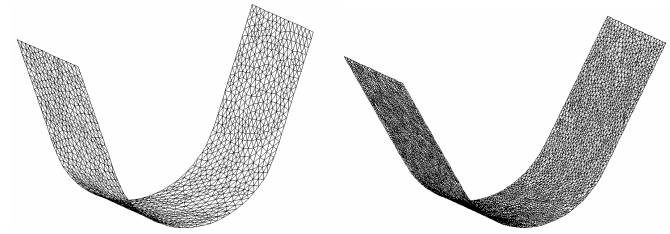

Figure 19. Irregular meshes for sheet geometry

\subsubsection{Regular meshes}

The reference solution for the shell analysis using Abaqus/Standard has been analyzed with a small element size $(0.25 \mathrm{~mm})$. The applied element size corresponds to a ratio $0.25 / 23.5=0.001$ for the punch and $0.25 / 4=0.06$ for the element size versus tool radius. The effect of increasing the element size on the calculated load displacement curve is displayed in Figure 20.

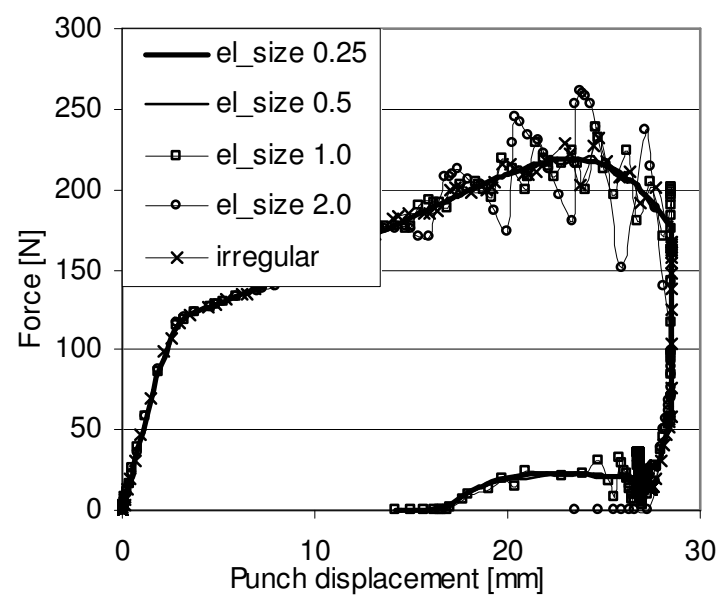

Figure 20. Effect of element size on load displacement curve

The fact that contact is only checked for a row of nodes of the sheet is clear from these figures. With increasing element length, the oscillations in the curve become more pronounced. Making the mesh irregular by shifting some nodes clearly results in a smoothening effect on the load displacement curve. Using larger elements also has a 
destabilizing effect on the convergence behavior (in particular in the unloading phase). The characteristic forming angles of these simulations are listed in Table 15.

\begin{tabular}{|l|l|l|}
\hline Element size $(\mathrm{mm})$ & forming angle & springback angle \\
\hline 0.25 & 21.83 & 53.89 \\
0.5 & 21.84 & 53.90 \\
1.0 & 21.77 & 53.46 \\
2.0 & 22.75 & no conv. \\
2.0 irregular & 21.79 & no conv. \\
\hline
\end{tabular}

Table 15. Variation of element size (Abaqus/Standard; $\mu=0.1348 ;$ shell)

Increasing the elements sizes from $0.25 \mathrm{~mm}$ to $1 \mathrm{~mm}$ reduces the characteristic angles. The difference in forming angle is $0.06^{\circ}$ and release angle is $0.43^{\circ}$. It is clear from the load displacement curve that this increase in element length destabilizes the solution procedure due to the local character of the contact in the bending process.

\subsubsection{Lateral bending effects}

Besides a curvature in the XY-plane (see Figure 3 for definition of XY-plane), also a lateral curvature of the sheet develops during bending; so called anticlastic bending. The influence of this lateral bending has been analyzed by varying the number of elements over the width of the sheet. The results, obtained by a shell model with 11 layers, using MSC.MARC and an instantaneous tool release (see section 4.7), are summarized in Table 16 and Table 17.

\begin{tabular}{|c|l|l|}
\hline lateral discretization & forming angle & springback angle \\
\hline 6 & 20.420 & 54.440 \\
12 & 20.422 & 54.432 \\
14 & 20.427 & 54.382 \\
\hline
\end{tabular}

Table 16. Variation in lateral sheet discretization (MSC.MARC; $\mu=0.1348$; shell)

\begin{tabular}{|c|l|l|}
\hline lateral discretization & forming angle & springback angle \\
\hline$* *$ & 20.50 & 45.20 \\
6 & 20.43 & 44.04 \\
12 & 20.43 & 45.46 \\
\hline
\end{tabular}

Table 17. Variation in lateral sheet discretization (MSC.MARC; $\mu=0.0$; shell). Plane strain results listed for comparison (**).

It has been observed that the lateral bending gives rise to a non-uniform distribution of plastic strain across the width. When looking at the results in case of friction, it can be concluded that the lateral discretization does not affect the simulation results. However, in case of the frictionless simulations, a difference of $1.5^{\circ}$ appears when using 6 elements instead of 12. An elaborated explanation for this difference is presented in section 6 . 


\subsection{Integration points over the thickness}

The bending stiffness of the sheet is numerically obtained by an integration scheme over the thickness. The number of integration points and applied integration scheme has been varied with Abaqus/Standard, Dieka and MSC.MARC. The results of simulations performed with Abaqus/Standard and Dieka (both using Gauss integration) are listed in Table 18 and Table 19, respectively. Note, that an odd number of integration points leads to an underestimation of the bending stiffness while an even number overestimates the bending stiffness. Increasing the number of integration points better approximates the correct bending stiffness.

\begin{tabular}{|l|l|l|}
\hline i.p.'s & Forming angle & springback angle \\
\hline 5 & 20.92 & 53.37 \\
7 & 20.95 & 53.40 \\
9 & 20.90 & 53.05 \\
11 & 20.92 & 52.90 \\
13 & 20.90 & 52.91 \\
\hline
\end{tabular}

Table 18. Variation in integration points (Abaqus/Standard; $\mu=0.1348$; shell)

\begin{tabular}{|l|l|l|}
\hline i.p.'s & Forming angle & springback angle \\
\hline 2 & 21.95 & 61.50 \\
3 & 21.98 & 54.13 \\
4 & 21.86 & 58.16 \\
5 & 21.95 & 56.42 \\
6 & 21.86 & 57.73 \\
7 & 21.93 & 56.96 \\
\hline
\end{tabular}

Table 19. Variation in integration points (Dieka; $\mu=0.1348 ;$ shell)

The default method for thickness integration of shell elements in MSC.MARC is Simpson's rule. Changing the number of integration points yields a variation in springback as given in Table 20 .

\begin{tabular}{|l|l|l|}
\hline i.p.'s & forming angle & springback angle \\
\hline 3 & 20.668 & 46.614 \\
5 & 20.402 & 54.360 \\
7 & 20.450 & 54.014 \\
9 & 20.4 & 54.7 \\
11 & 20.422 & 54.476 \\
\hline
\end{tabular}

Table 20. Variation in integration points (MSC.MARC; $\mu=0.1348$; shell)

In view of the considerable deviation when just 3 layers are used, it appears that 5 layers is the recommended minimum in this case. The number of integration points 
across the thickness, if higher than 5, was seen to have no effect on the force displacement curve.

\subsection{Tool release method}

A springback simulation can be performed by gradually retracting the punch. However, this method of unloading is computational costly. Therefore, in most springback analyses, the response of the sheet due to the release of the tools is numerically performed in one increment, a so-called instantaneous release. Sometimes this increment is subdivided into a number of sub increments in order to avoid numerical instabilities. These occur because the contact forces are suddenly removed and transformed to residual forces. If these residual forces are removed suddenly, this can easily result in local buckling effects and hence this is sometimes done in a number of steps. In this section both release methods will be compared in case friction and no friction is applied, using Abaqus/Standard and MSC.MARC. 2D simulations are performed with plane strain elements as well as 3D simulations with shell elements. The results of plane strain simulations are listed in Table 21, Table 22 and Table 23; the results of the shell simulations are listed in Table 24 and Table 25. Note that there is a difference in forming angle when using Abaqus/Standard or MSC.MARC (Table 22 and Table 23), which is due to the difference in contact description, see section 4.1 and 4.2.

\begin{tabular}{|l|l|l|}
\hline release method & Forming angle & springback angle \\
\hline Gradual & 20.48 & 45.89 \\
Instantaneous & 20.48 & 45.89 \\
\hline
\end{tabular}

Table 21. Variation in release method (MSC.MARC; $\mu=0.0$; plane strain)

\begin{tabular}{|l|l|l|}
\hline release method & Forming angle & springback angle \\
\hline Gradual & 20.46 & 54.11 \\
Instantaneous & 20.46 & 55.67 \\
\hline
\end{tabular}

Table 22. Variation in release method (MSC.MARC; $\mu=0.1348$; plane strain)

\begin{tabular}{|l|l|l|}
\hline release method & Forming angle & springback angle \\
\hline gradual & 21.84 & 54.45 \\
Instantaneous & 21.84 & 56.44 \\
\hline
\end{tabular}

Table 23. Variation in release method (Abaqus/Standard; $\mu=0.1348$; plane strain)

\begin{tabular}{|l|l|l|}
\hline release method & Forming angle & springback angle \\
\hline gradual & 20.89 & 43.30 \\
Instantaneous & 20.89 & 43.35 \\
\hline
\end{tabular}

Table 24. Variation in release method (Abaqus/Standard; $\mu=0.0 ;$ shell) 


\begin{tabular}{|l|l|l|}
\hline release method & Forming angle & springback angle \\
\hline gradual & 20.92 & 53.27 \\
instantaneous & 20.92 & 55.26 \\
\hline
\end{tabular}

Table 25. Variation in release method (Abaqus/Standard; $\mu=0.1348 ;$ shell)

According to these results, there is no difference between both release methods in the frictionless situation. However, if friction is taken into account, the springback angle can vary up to $2^{\circ}$ degrees depending on whether the strip is released instantaneously or gradual. During the forming stage tangential forces are present on the sheet due to the friction. Tool removal will result in a release of the tangential forces before the bending moment is decreased. This causes modified contact, whereas an instantaneous release does not account for this sequential effect.

The different response of the gradual and instantaneous unloading procedures is probably linked to a slight increase of the plastic deformation during unloading. To investigate this, the equivalent plastic strain along the top of the sheet is presented in Figure 21 as a function of the current arc length along the sheet. Since the sheet is symmetric, only the strain distribution of the right part of the sheet is reported, meaning that the origin of Figure 21 corresponds to the center position of the punch. Graphs are given for several stages during forming (till $1.0 \mathrm{sec}$.) and several stages during springback (till $1.1 \mathrm{sec}$.).

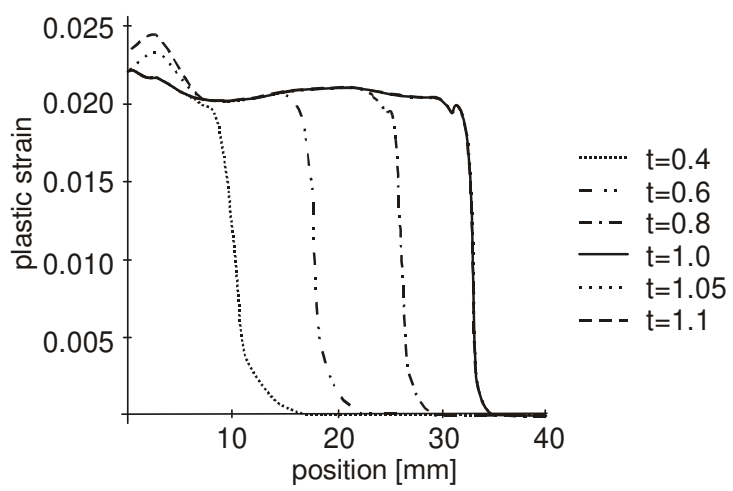

Figure 21. Evolution of equivalent plastic strain along the top of the sheet.

From inspection of the plastic strain distribution along the top of the strip, it appears that the plastic strain near the punch center starts to increase as the unloading starts. Almost immediately upon retracting the punch, the tangential force (friction) is reversed. Instead of a global tensile force superimposed upon the bending moment a global compressive force develops. This can happen because contrary to the tangential force the normal force in contact needs a finite displacement to decrease to 0. Since the inner 
radius of the sheet is in compression due to bending, adding a compressibe force will promote further plastic straining upon unloading. This local plastic bending continues for app. $0.1 \mathrm{~s}$. No change of plastic strain has been observed for the instantaneous procedure, which therefore results in a purely elastic unloading.

Since the gradual release method is supposed to be more realistic, it is concluded that instantaneous unloading can lead to a substantial error. A gradual unloading method is therefore required for this phenomenon to be captured properly. Unfortunately the gradual release is numerically very sensitive to load stepping and a high amount of small time steps is needed.

\subsection{Material model}

In this section, the influence of the used material model on the angles after forming and after springback is investigated. Simulations are performed with both the FE packages MSC.MARC and Dieka. First the results of the MSC.MARC simulations are focused on. One simulation is performed using the Von Mises (isotropic) material model. Three simulations are performed, using the Hill'48 planar anisotropic material model, in which the rolling direction is varied. The R-values of the aluminum sheet used for the Numisheet 02 benchmark will be used $\left(R_{0}=0.894, R_{45}=0.611, R_{90}=0.660\right)$. The results of these simulations are listed in Table 26.

\begin{tabular}{|l|l|l|}
\hline material type & Forming angle & springback angle \\
\hline von Mises & 20.5 & 42.7 \\
Hill'48 (0) & 20.5 & 40.6 \\
Hill'48 (45) & 20.5 & 38.7 \\
Hill'48 (90) & 20.5 & 37.7 \\
\hline
\end{tabular}

Table 26. Variation in material model (MSC.MARC; $\mu=0.0$; shell)

Table 26 shows that the angle after forming is insensitive to the material model employed. However, introducing anisotropy in the material model, gives rise to large deviations in the angle after springback, despite the use of an identical Young's modulus and initial yield stress. Considering the minor difference in strain hardening, the difference in springback angle has to be wholly attributed to the shape of the yield function, which gives rise to a different yield stress.

Next, the results, gained with Dieka are treated. In this code, the Vegter material model is available, amongst the Von Mises and Hill'48 material model. The major advantage of the Vegter material model over the Hill' 48 material model is it is based on data from multi-axial stress states, i.e. the pure shear point, the uni-axial point, the plane strain point and the equi-bi-axial point, see Figure 22. The yield surface is constructed 
using Bezier interpolations, to ensure a smooth yield function (Vegter, 2004). Since the Hill'48 material model is based on the uni-axial tensile data only, the Vegter yield function is better capable of describing the material behavior, especially in case of aluminum (Boogaard, 2002), (Pijlman, 2001).

\section{Figure 22. Vegter yield locus}

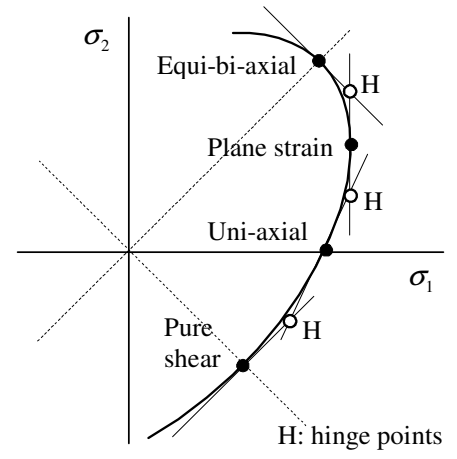

In order to make a proper comparison between the available yield functions, the Rvalues of the aluminum sheet used for the Numisheet 02 benchmark will be used $\left(\mathrm{R}_{0}=\right.$ $\left.0.894, R_{45}=0.611, R_{90}=0.660\right)$. The results of the performed simulations are listed in Table 27.

\begin{tabular}{|c|c|c|}
\hline Material model & forming angle & springback angle \\
\hline Von Mises & 21.94 & 56.58 \\
Hill & 21.96 & 58.12 \\
Vegter & 21.97 & 56.33 \\
\hline
\end{tabular}

Table 27. Variation in material model (Dieka; $\mu=0.1348$; shell)

Also from these results, it can be concluded that using different material models has a large influence on the springback angle; the difference between the Hill' 48 yield function and the Vegter yield function is $1.7^{\circ}$. However, this could be expected in case of using aluminum, since the Hill' 48 yield function is not capable to accurately describe the plastic deformation of material having R-values lower than 1 (Boogaard, 2002). Besides, the results of the Von Mises model are closer to the Vegter model than to the Hill' 48 model, which is in line with earlier experiences. However, more research in this field is recommended. 


\section{Sensitivity analysis: Physical factors}

\subsection{Friction coefficient}

The benchmark problem has been analyzed both without friction and with a Coulomb friction model where the friction coefficient was set to 0.1348 , according the Numisheet 2002 specifications. Simulations are performed with Abaqus/Standard, MSC.MARC and Dieka.

Note, that the 'original' Coulomb friction model is a true stick-slip model, whereas often a regularized variant is implemented in finite element codes, which reflects the elastic stiffness (to improve the stability of the calculation). All simulation results, reported here are performed with the defaults, as set by the specific code itself.

First the results of Abaqus/Standard are treated. A 2-D plane strain finite element model was used. The characteristic values of the angles at the end of the forming stage and after springback are listed in Table 28.

\begin{tabular}{|l|l|l|}
\hline$\mu$ & forming angle & springback angle \\
\hline 0 & 21.88 & 48.38 \\
0.1348 & 21.84 & 54.45 \\
\hline
\end{tabular}

Table 28 Variation in friction coefficient (Abaqus/Standard; shell)

The characteristic load displacement curves for the analysis without friction and with friction are shown in Figure 23. 


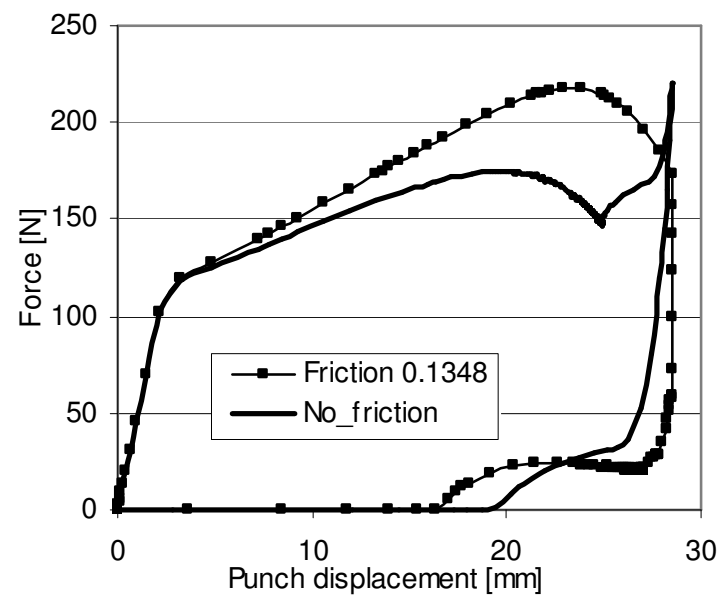

Figure 23. Load displacement diagram plane strain analysis for $\mu=0$ and $\mu=0.1348$

Note that the unloading stage is analyzed by a physical retraction of the punch. A high number of steps are required for this unloading (in particular for the problem with friction; more steps are required for the unloading than the loading phase). From these results it can be concluded that friction has a large influence on the springback behavior. However, we have to be careful with drawing conclusions, since the force displacement diagrams show a different shape, which indicates a different mode of deformation. Therefore a closer look is taken in the deformation history of the sheet.

In case of the simulation with friction, the tangential forces due to friction result in a stretching of the sheet, causing contact between punch and the sheet to be present at the complete forming stage. During forming, the contact spot increases along the punch. As a result, the bending arm decreases, and since the moment is more or less constant, the punch force increases. Contrary, moving the punch downwards, geometrically means that the moment arm will increase. If the latter effect dominates the effect of the moving contact spot, the punch force will decrease, as can be observed after a punch stroke of \pm 24 [mm], see Figure 23 .

In case of the simulation without friction, the former explanation also applies. However, since friction is absent, the required punch force is lower (when friction applies, energy is dissipated which has to be supplied by an increase of the punch force, since the punch stroke is constant) and the maximum is reached at a lower punch stroke. During forming the sheet separated at the lower section of the punch. At \pm 25 [mm] the sheet gets in contact with the lower section of the die. As soon as this contact is present, 
the sheet is further bend into the final shape and the required force increases, see Figure 23.

Note: the release of the sheet from the punch center means that curvature increases beyond punch curvature and thus the bending moment increases. That this happens nonetheless is probably due to the fact that contact pressure at the punch adds up with compressive bending stresses. This causes hydrostatic stress to increase and consequently effective stress to decrease. Since efective stress drives plastic deformation at the contact point plastic deformation is hindered somewhat.

The code Dieka is used to further investigate the influence of the friction coefficient, by varying it between 0 and 0.25 . The simulations are performed with shell elements and the results are listed in Table 29.

\begin{tabular}{|l|l|l|}
\hline$\mu$ & forming angle & springback angle \\
\hline 0 & 21.85 & 47.84 \\
0.01 & 21.82 & 51.40 \\
0.02 & 21.80 & 55.28 \\
0.03 & 21.80 & 57.05 \\
0.04 & 21.84 & 57.59 \\
0.05 & 21.89 & 57.33 \\
0.1 & 21.93 & 56.64 \\
0.1348 & 21.95 & 56.42 \\
0.2 & 21.95 & 56.11 \\
0.25 & 21.95 & 55.97 \\
\hline
\end{tabular}

Table 29. Variation in friction coefficient (Dieka; shell)

It can be concluded that increasing the friction coefficient from 0.04 till 0.25 yields a decrease in the angle after springback of $1.6^{\circ}$. However, the angle after springback largely depends on the friction coefficient in the region from 0 to 0.03 . The reason for this is already explained by the difference in deformation history, for a friction coefficient of 0.03 and less, the sheet doesn't follow the punch shape but makes a sharper bending radius (which increases with decreasing friction coefficient). This phenomenon has a large influence on the springback behavior. Note that in practice, the friction coefficient can hardly be defined within a range of 0.03 . This would mean that a small mismatch of the friction coefficient will lead to useless springback predictions. However, the case at hand is a specific case (single curved product), and is not representative for real deep drawing cases.

Finally, the code MSC.MARC is used to investigate the influence of the friction coefficient, by varying it between 0 and 0.1348 using, a 2D plane strain model. The results are presented in Table 30 . 


\begin{tabular}{|l|l|l|}
\hline$\mu$ & forming angle & springback angle \\
\hline 0.0 & 20.497 & 45.204 \\
0.025 & 20.501 & 54.763 \\
0.05 & 20.492 & 53.876 \\
0.10 & 20.474 & 53.638 \\
0.1348 & 20.461 & 52.905 \\
\hline
\end{tabular}

Table 30. Variation in friction coefficient (MSC.MARC; plane strain)

These results show the same trend as found in the former investigations; for very low friction coefficients the angle after springback drastically increases, while for higher friction coefficients the springback angle slowly decreases, see Figure 24.

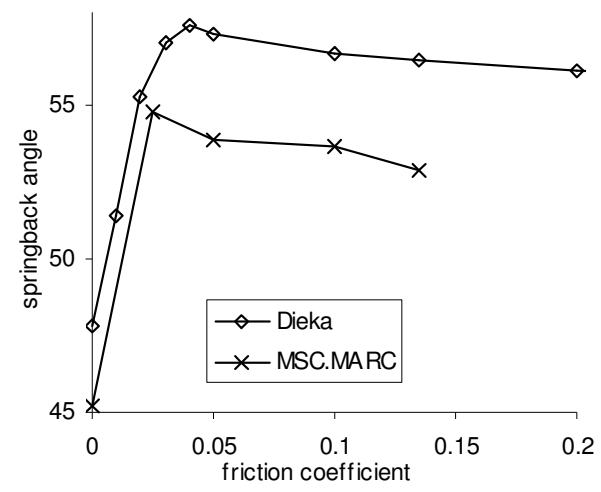

Figure 24. Springback angle as a function of the friction coefficient

\subsection{Punch speed}

In this section, the influence of the punch speed on the simulation results is investigated, when using an explicit time algorithm. The finite element code used in this section is Optris.

Four simulations are performed in which the punch velocity is set to $0.5 \mathrm{~m} / \mathrm{s}, 1 \mathrm{~m} / \mathrm{s}, 2$ $\mathrm{m} / \mathrm{s}$ and $4 \mathrm{~m} / \mathrm{s}$, respectively. As the punch continuously touches the workpiece during forming, any acceleration of the punch will be partly transmitted to the sheet, which causes excitation of its dynamic eigen modes. To avoid high frequency vibrations of the sheet as much as possible, it is therefore important to change the punch speed gradually, so as to limit its acceleration and to reduce the contribution from the high frequency part 
of the velocity spectrum. For this purpose, the velocity profile shown in Figure 25 has been selected. The velocity profile shown corresponds to the slowest punch motion considered with a maximum velocity of $0.5 \mathrm{~m} / \mathrm{s}$. For the other cases, the same profile but a different scale along the y-axis, has been used.

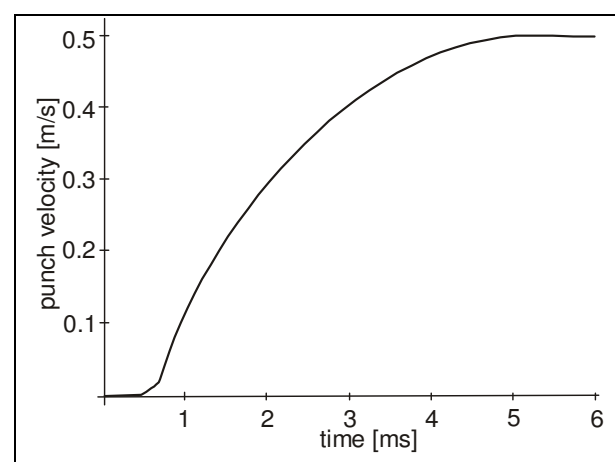

Figure 25. Gradual variation of punch velocity as a function of the time

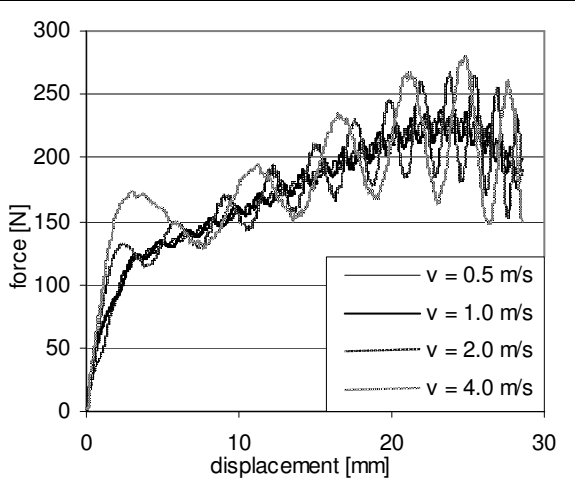

Figure 26. Force-displacement curve of for different punch velocities.

Note that in all cases the maximum of the curve is reached before completion of the forming stage, since the simulation with a punch speed of $0.5 \mathrm{~m} / \mathrm{s}$ takes $57 \mathrm{~ms}$ for the forming stage whereas the simulation with a punch speed of $4 \mathrm{~m} / \mathrm{s}$ takes $7.2 \mathrm{~ms}$ for the forming stage. Note that this is a conservative estimate of the effects, because one could also set the maximum speed to be reached to a fixed punch displacement, e.g. 10\%. This would lead to larger accelerations than the present choice. The springback angles obtained for the different punch velocities are given in Table 31. The corresponding force-displacement curves are represented in Figure 26.

\begin{tabular}{|l|l|l|}
\hline punch speed $(\mathrm{m} / \mathrm{s})$ & forming angle & springback angle \\
\hline 0.5 & 20.50 & 56.635 \\
1.0 & 20.50 & 56.631 \\
2.0 & 20.53 & 56.624 \\
4.0 & 20.49 & 56.690 \\
\hline
\end{tabular}

Table 31. Variation punch speed (Optris: $\mu=0.1348$ : shell)

It can be concluded that the punch speed has only a minor effect on the angle after springback $\left(0.2^{\circ}\right)$. However, the effect on the force-displacement curve of the punch is significant, the shape of the curve changes dramatically with increasing punch speed. It 
seems that the curve can be decomposed into a quasi-static response given by a smooth curve, and a periodic disturbance with almost sinusoidal shape. Clearly the amplitude of this disturbance increases with the punch speed. At first sight it seems that the frequency of the signal reduces as the punch speed increases. However, since the time period corresponding to the total punch displacement in case of $1.0 \mathrm{~m} / \mathrm{s}$ is about four times as large as that in case of $4.0 \mathrm{~m} / \mathrm{s}$, it can be seen that the frequency is similar for all cases. However, one has to be careful with using higher punch speed scaling. In general, explicit codes make use of significant mass scaling (or speed scaling), an more research in this field is recommended.

\subsection{Hardening properties}

The input for a material model in a finite element code is gained by experimental testing of the used material. Generally the material is tested by uni-axial tensile tests. However, the material properties do vary within a coil and, more importantly, between coils. To investigate the influence of variation in material properties on the springback behavior, different material parameters will be varied. In this section, the extended Ludwik Nadai hardening law is used (equation ( 2 )) in combination with the Hill'48 yield criterion.

$$
R_{p}=C\left(\varepsilon_{0}+\varepsilon_{p}\right)^{n} \text { with } \varepsilon_{0}=\left(\frac{R_{p 0}}{C}\right)^{\frac{1}{n}}
$$

where $R_{p}$ is the yield stress, $\varepsilon_{p}$ is the equivalent plastic strain, $C$ is the hardening coefficient and $n$ the hardening exponent. The parameter $\varepsilon_{0}$ can be seen as a pre-strain. For the default material properties, the value $\varepsilon_{0}$ equals $8.12 \mathrm{e}^{-3}$. Three sets of simulations are performed with Dieka, in which the yield stress, the hardening coefficient and hardening exponent are varied. The yield stress is varied with $\pm 10 \%$ deviation, where the hardening coefficient and hardening exponent are kept constant. The reason for choosing $10 \%$ deviation is that the initial yield stress is difficult to measure. The hardening coefficient is varied with $\pm 10 \mathrm{MPa}$, the hardening exponent is varied with $\pm 5 \%$ deviation. In both latter situations, the initial yield stress is kept constant which means that the pre-strain $\varepsilon_{0}$ is implicitly varied. The influence of the variation in material parameters on the stress-strain curve is visualized in Figure 27. A zoom in of this figure in the plastic strain range $0-0.05$ is presented in Figure 28. 


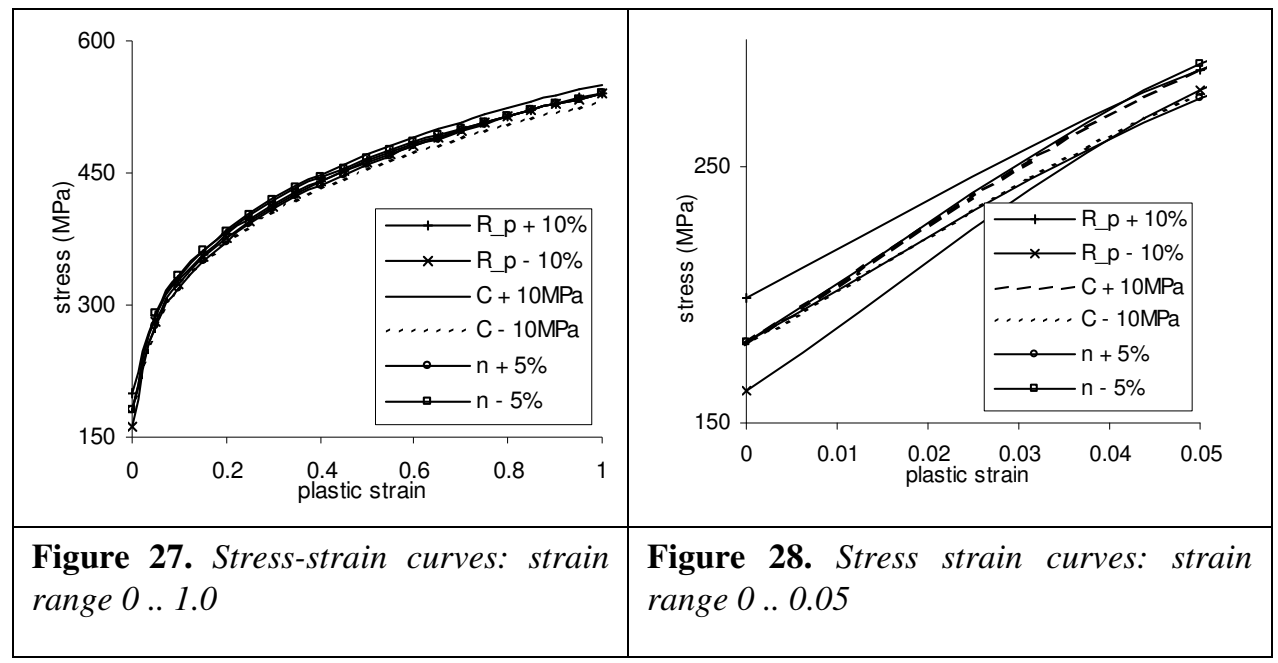

The influence of the variation in material parameters on the angle after forming and after springback is summarized in Table 32, Table 33 and Table 34.

\begin{tabular}{|l|l|l|}
\hline$\sigma_{\mathrm{y} 0}(\mathrm{MPa})$ & forming angle & Springback angle \\
\hline 199.98 & 21.98 & 57.76 \\
181.80 & 21.95 & 56.42 \\
163.62 & 21.90 & 55.28 \\
\hline
\end{tabular}

Table 32. Variation in yield stress (Dieka; $\mu=0.1348$; shell)

\begin{tabular}{|l|l|l|}
\hline$C(\mathrm{MPa})$ & forming angle & Springback angle \\
\hline 549.5 & 21.94 & 56.82 \\
539.5 & 21.95 & 56.42 \\
529.5 & 21.96 & 55.99 \\
\hline
\end{tabular}

Table 33. Variation in hardening coefficient (Dieka; $\mu=0.1348 ;$ shell)

\begin{tabular}{|l|l|l|}
\hline$N$ & forming angle & Springback angle \\
\hline 0.2373 & 21.97 & 55.65 \\
0.226 & 21.95 & 56.42 \\
0.2147 & 21.93 & 57.27 \\
\hline
\end{tabular}

Table 34. Variation in hardening exponent (Dieka; $\mu=0.1348$; shell)

A deviation of $10 \%$ in yield stress causes a maximum spread of $2.5^{\circ}$ in the angle after springback; a deviation of 5\% in hardening exponent causes a maximum spread of $1.6^{\circ}$ in the angle after springback. However, the unconstrained bending problem is less sensitive for changing the Hardening coefficient. The different levels of sensitivity can be explained when focusing on the strain distribution in the sheet. The maximum plastic 
strain appearing in the sheet is approximately $2 \%$. Figure 28 shows a large deviation in flow curves when varying the initial yield stress and hardening exponent in the range of low plastic strains. In this range, the flow curve is less sensitive for variations in the Hardening coefficient. However, note that for large strains, the flow curve is more sensitive for variations in Hardening coefficient and less sensitive for variations in hardening exponent and initial yield stress.

\subsection{Lankford anisotropy parameter}

As stated before, material properties do vary within a coil and between different coils. In this section, the influence of variation of the Lankford parameter $\mathrm{R}$ on the springback behavior will be investigated. The material will be assumed planar isotropic, meaning that the R-value for the $0^{\circ}, 45^{\circ}$ and $90^{\circ}$ is equally set to 0.894 . A variation of $\pm 5 \%$ is be applied and the results are listed in Table 35 . It can be seen that a variation in $\mathrm{R}$-value hardly influences the angle after springback for both test problems, likely due to the low level of plastic deformation.

\begin{tabular}{|l|l|l|}
\hline R-value & forming angle & Springback angle \\
\hline 0.849 & 21.96 & 56.32 \\
0.894 & 21.95 & 56.42 \\
0.939 & 21.95 & 56.48 \\
\hline
\end{tabular}

Table 35. Variation in R-value (Dieka; $\mu=0.1348$; shell)

\subsection{Sheet thickness}

\section{Variation in uniform sheet thickness}

In practice, the sheet thickness within a coil and between coils will show some variation. In this section the influence of a variation in the sheet thickness is investigated. Two sets of simulations will be performed, i.e. in one set the uniform sheet thickness is varied, in the second set the sheet thickness homogeneity is varied.

According the DIN-standard, the sheet thickness is allowed to show a deviation of $10 \%$. Three simulations are performed, varying the uniform sheet thickness from $0.9 \mathrm{~mm}$ to $1.1 \mathrm{~mm}$. The results of the simulation are listed in Table 36 . 


\begin{tabular}{|l|l|l|}
\hline Sheet thickness & Forming angle & springback angle \\
\hline 0.9 & 23.01 & 60.74 \\
1.0 & 21.95 & 56.42 \\
1.1 & 20.37 & 52.14 \\
\hline
\end{tabular}

Table 36. Variation in uniform sheet thickness (Dieka; $\mu=0.1348$; shell)

It can be seen clearly that varying the thickness with $10 \%$ has a large influence on the springback angle. Note that in the current state of steel making, the variation of the sheet thickness between coils is less than $10 \%$ in general, which makes the difference in springback angle less pronounced.

Second, the influence of variation in sheet thickness homogeneity is investigated. In Dieka, the initial sheet thickness is given as a nodal value. At the start of the simulation these nodal values are interpolated to the integration points. A random variation of $\pm 2.5 \%$ (sheet thickness between 0.975 and 1.025) in initial sheet thickness homogeneity is prescribed and two different distribution patterns are set, sheet I and sheet II, respectively. In sheet $I$, the sheet is assumed to be the thinnest at all edges, whereas the thickness in the inner part is randomly distributed. In case of sheet II, the entire sheet is randomly distributed. The simulation results are listed in Table 37.

\begin{tabular}{|l|l|l|}
\hline & forming angle & springback angle \\
\hline Type 1 & 21.87 & 56.07 \\
Type 2 & 21.97 & 56.17 \\
Uniform & 21.95 & 56.42 \\
\hline
\end{tabular}

Table 37. Variation in sheet thickness homogeneity (Dieka; $\mu=0.1348$; shell)

A variation in the sheet thickness homogeneity hardly influences the springback behavior in both test problems. It is expected that a variation in the sheet thickness homogeneity only play a role in case of the initiation of local necking.

\section{Sensitivity analysis: 2D vs. 3D modeling}

The simplicity of the tool and sheet geometry assumes the problem to be considered as $2 \mathrm{D}$ plane strain. However, this will result in neglecting the lateral bending effects. The consequence of this neglect will be demonstrated in this section. A plane strain versus shell approach comparison has been done for penalty contact (equivalent numerical value $1000 \mathrm{MPa} / \mathrm{mm}$ ) for both the case with and without friction. The load displacement curves are shown in Figure 29 and Figure 30, respectively. 


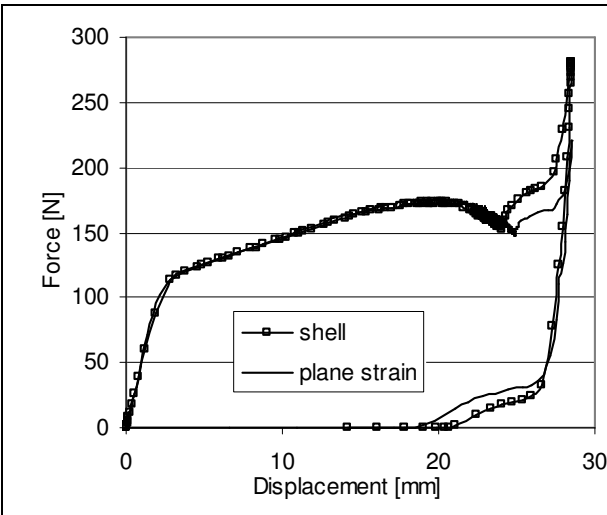

Figure 29. Comparison plane strain versus shell analysis $(\mu=0.0)$

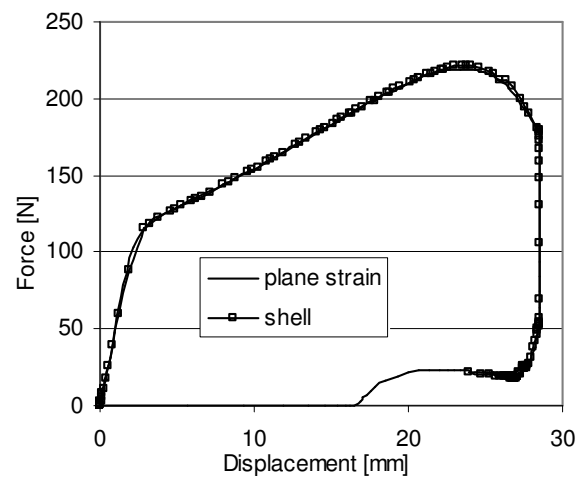

Figure 30. Comparison plane strain versus shell analysis $(\mu=0.1348)$

\begin{tabular}{|l|l|l|}
\hline & forming angle & springback angle \\
\hline Plane strain & 20.94 & 47.69 \\
Shell & 20.89 & 43.30 \\
\hline
\end{tabular}

Table 38. Comparison of angles plane strain and shell approach (Abaqus/Standard; $\mu=0.0)$

\begin{tabular}{|l|l|l|}
\hline & forming angle & springback angle \\
\hline Plane strain & 20.90 & 53.55 \\
Shell & 20.92 & 53.27 \\
\hline
\end{tabular}

Table 39. Comparison of angles plane strain versus shell (Abaqus/Standard: $\mu=0.1348$ )

The resulting values of the characteristic angles are listed in Table 38 and Table 39. In case of friction a difference of $0.02^{\circ}$ is present in the forming angle whereas the difference after springback is $0.28^{\circ}$. However, in case of the frictionless situation, the difference between the plane strain and sheet formulation is severe. Here, the deviation amounts $4.4^{\circ}$. In the frictionless simulations, contact between the punch and the sheet is lost at a certain punch stroke, see section 5.1. Hence, the anticlastic bending effects are not restricted due to the punch, and therefore its influence can be clearly observed in springback angle. The anticlastic bending effects in the analysis with friction are less pronounced since the contact between punch and sheet and punch is preserved during the simulation. Concluding, the authors recommend not using plane strain analyses for these kinds of deformation processes. 


\section{Conclusions}

Note: A 'large influence' in this chapter means a scatter larger than $2^{\circ}$ in springback angle. This is based on the fact that in practice an angle deviation of more than $2^{\circ}$ is deemed unacceptable.

It is remarkable that a much larger scatter is found in the Numisheet 2002 benchmark B (typically within the range 45 to $72^{\circ}$ ), than in the sensitivity analysis at hand (typically in the range 44 to 56, with a large effect caused by the application of zero friction). The Numisheet benchmark showed that the analyst plays an important role in the simulation process. The unrealistically large scatter in the benchmark results $\left( \pm 25^{\circ}\right)$ are likely caused by inexperienced users.

The applied method for contact and the applied numerical values of the penalty stiffness determines the numerical stability and the obtained accuracy. An unrealistic choice of contact or tool stiffness can have a large influence on the springback behavior. Low values of the penalty factor have a smoothing effect on the solution, high values of the penalty stiffness cause convergence problems.

Numerical damping techniques can be applied to stabilize the computation. However, since damping has a large influence on the forming process and the springback behavior, its use has to be minimized in a numerical simulation and checked against the dissipated work by damping.

The friction coefficient has a large effect on the springback behavior of this component in case of a low friction coefficient. The reason of this is a difference in deformation behavior in case a high or a low friction coefficient is applied. The influence of the friction coefficient on the springback behavior decreases for increasing friction coefficient (similar deformation behavior). For a proper sensitivity study on the influence of the friction (friction coefficient, friction models, contact pressure etc) on the springback behavior, further investigation on other components is necessary.

The method of unloading, i.e. direct release (omitting all tools) versus gradual release (physical retraction of the punch) has a large effect on the springback behavior. In the latter method, springback is not necessarily entire elastic.

The number of integration points through the sheet thickness has a large effect on the springback behavior. At least 5 integration points through the sheet thickness are necessary to be able to properly describe the springback phenomenon. To accurately describe spring-back, more integration points are necessary through the sheet thickness.

The element size has a large influence on the springback prediction. Too coarse a mesh influences the approximation of local contact phenomena, lateral bending and spatial discretization, which all have its influence on the springback behavior. 
Anticlastic bending, and thus structural stiffness, has a large influence on the springback behavior. Degeneration of 3D problems to 2D problems is therefore not an option when performing this type of springback simulations.

A variation in sheet thickness homogeneity hardly influences the springback behavior. A variation in the uniform thickness can have a large influence on the springback behavior, provided that the variation is as large as the DIN-norm allows.

Springback behavior can be sensitive for variations in material properties, especially for products with low level of plastic deformation. The influence drastically decreases with an increasing strain level.

The importance of the material behavior is seen through the dependence of the springback results on the applied yield function (anisotropy).

The results of the Numisheet 2002 benchmark and the sensitivity analyses do not confirm that springback cannot be handled by explicit integration methods.

Since the unconstrained bending problem is a numerically very sensitive one, care has to be taken to transfer the results obtained by analyzing this problem to other components. Besides, the bending effects clearly dominate the unconstrained bending problem and as a consequence finite element programs or technologies, which treat bending effects in an approximate way, are not very successful in predicting the correct behavior for this component.

\section{Recommendations}

Unexpectedly, the method of unloading has a large influence on the springback behavior. In case of gradual release, the plastic strain increases after springback. However, a disadvantage of the gradual release method is that is very expensive with respect to computation time, in particular do to the difficult convergence behavior of friction at low values of the normal forces. Research has to be done, either on the improved numerical stability of friction or on replacing the direct release by a 2-step release, i.e. first a release of the tangential force, second the release of the normal force. It is expected that this type of release approximate the gradual release at low computational costs.

A large number of integration points through the sheet thickness yields more accurate results. However, it also increases the computational costs. In regions without a neutral line in the stress distribution through the thickness, less integration points through the thickness are needed compared to regions with a neutral line. A suggestion can be to investigate the possibility of making the number of integration points variable on the curvature or through thickness stress gradient. This calls for accurate mapping algorithms for integration point data, when the number of integration points changes 
during the computation. Also the use of a fixed number of integration points through the sheet thickness in which the position of the integration points can be varied (to accurately capture the transition from elastic to plastic behavior) seems worthwhile to investigate.

Sensitivity analyses of a variation in material properties and sheet thickness on the springback behavior are performed. However, the values used in these analyses are not based on real data. Research has to be done on the levels of deviation of the material properties and sheet thickness. The conclusions, drawn from these analyses, have to be adapted, based on the results of this research.

In general, simulations have to be performed by experienced users. Proper guidelines for accurate springback simulations will help to relax this requirement.

\section{Acknowledgement}

This research was carried out under project number MC1.02121 in the framework of the Strategic Research programme of the Netherlands Institute for Metals Research in the Netherlands (www.nimr.nl).

\section{References}

Numisheet 2002, Proceedings of the $5^{\text {th }}$ International Conference on Numerical Simulations of 3-D sheet Metal Forming Processes, D-Y. Yang et al. (eds.), Jeju Island, Korea, 2002, www.numsiheet2002.org

Batoz J.L., P. Lardeur, A discrete shear triangular nine d.o.f. element for the analysis of thick to very thin plates, IJNME, vol. 28, p. 533-560, 1989

Optris manual, 2002

MSC.MARC manual, 2002

Boogaard van den, A.H., Thermally enhanced forming of aluminium sheet, Ph.D. Thesis, University of Twente, 2002

Pijlman H.H., Sheet material characterisation by multi-axial experiments, Ph.D. Thesis, University of Twente, 2001

Vegter H., A.H. van den Boogaard, A yield function for anisotropic sheet material by interpolation of biaxial stress states, submitted to: International Journal of Plasticity, 2004 